\title{
Communicatio Socialis
}

ZEITSCHRIFT FÖR PUBLIZISTIK IN KIRCHE UND WELT

In Verbindung mit

Michael Schmolke (Münster), Karl R. Höller (Aachen)

und Kees Verhaak (Nimwegen)

herausgegeben von

Franz-Josef Eilers svd (Rom/Genf)

in Zusammenarbeit mit der Gesellschaft Katholischer Publizisten

Deutschland e.V.

\section{Am Beispiel Ceylon:}

Die Rolle der Kirche in der Presse der Entwicklungsländer von Joe Nethising be

Die Geschichte der weltlichen wie der religiösen Presse in Ceylon reicht in ihren Anfängen mehr als hundert Jahre zurück.

Im weltlichen Bereich sind die englischsprachigen Abendzeitungen zu nennen: „Ceylon Observer", gegründet 1834, und: "Times of Ceylon", 1846. „Ceylon Daily News ${ }^{\alpha}$, die größte englische Morgenzeitung, wurde 1918 gegründet.

Bei der religiösen Presse: die katholische „Gnanartha Pradipaya“, 1866, das älteste bestehende Sinhala-Wochenblatt; "Messenger ", 1869, die einzige katholische englische Wochenzeitung.

Bei jeder Wertung der ceylonesischen Presse und ihrer Rolle muß dem SinhalaWochenblatt "Sulumina" besondere Beachtung geschenkt werden, weil es eine einzigartige Stellung in der östlichen Zeitungswelt einnimmt. Seine Auflage ist die höchste im gesamten Nahen Osten.

Wie die Bedeutung der englischen Presse auf Ceylon unbestreitbar ist, so kann auch nicht geleugnet werden, daß eine massive asiatische Northcliffe-Revolution derzeit im Gange ist. Der Trend zur Massenpresse, wie er in England von Lord Northcliffe eingeleitet wurde, hat heute sein Gegenstück in der national orientierten SwabashaPresse in Ceylon wie auch in anderen asiatischen Entwicklungsländern.

P. Joe Nethisinghe ist Nationaldirektor für Massenmedien auf Ceylon, Leiter des „Social Communications Centre“ in Colombo und Vizepräsident von UNDA-Asien. 
Um den wachsenden Bedürfnissen der neuen Lesekundigen in Asiens Entwidklungsländern zu begegnen, müssen die asiatischen Zeitungen - nach Meinung der UNESCO - ihre gegenwärtigen Auflagen bis 1975 verdoppeln, um den Anforderungen auch nur einigermaßen gerecht $\mathrm{zu}$ werden. So steht die asiatische Presse vor der drängenden Aufgabe, die Forderungen einer rasch wachsenden Menge von neuen Lesekundigen nach Information zu erfüllen.

Für die Anforderungen, die die Rolle der Kirche in der Presse der Entwicklungsländer bestimmen, können wichtige Merkmale aus den Wünschen und Abneigungen der neuen Lesekundigen abgeleitet werden. Der Direktor des Asien-Programms vom International Press Institute - Tarzie Vittachi ${ }^{1}$ - hat folgende Charakteristika zusammengestellt:

- Die „neuen Leser“ werden voraussichtlich „ernsthafter “ sein als ihre westlichen Zeitgenossen.

- Sie werden in Denken, Geschmack und Einstellung weniger west-orientiert sein als die früheren Generationen asiatischer Zeitungsleser.

- Sie werden ein ausgeprägteres Bewußtsein für politische und wirtschaftliche Probleme haben als ihre westlichen Zeitgenossen, weil Menschen, die in einer allgemeinen Notlage leben, direkter mit Politik befaßt sind als die Menschen in den entwickelten Ländern.

- Sie werden sich immer mehr auf die "Sprachen"-Presse zubewegen, auf die Zeitungen in der Landessprache, als Quelle für Information, Führung und Unterhaltung.

- Sie werden zunehmend von rascher und müheloser Kommunikationstechnik angezogen werden: Hörfunk, Fernsehen, Bildpresse.

- Sie werden immer mehr Auskunft über ihren Staat, ihre Nation und ihre internationalen Beziehungen verlangen, und zwar in der hier genannten Rangfolge.

Daraus wird deutlich, daß sich für die Kirche in den Entwicklungsländern eine ganz neue Dimension erschließt: von einer christlichen Einstellung im Dienste der Nation her die katholische Presse zu sinnvolleren Zielen der Nachrichtenverbreitung, der Führung und Leitung der Massen zu bringen.

Die genaue Rolle der Kirche in der Presse der nächsten Jahre ist somit völlig klar: Die Kirche muß ihre Presseorgane auf strukturale Veränderung ausrichten, die der politischen, wirtschaftlichen und sozialen Lage des Entwicklungslandes entspricht und besser als zuvor den Geist christlicher sozialer Gerechtigkeit einschließt. Der Beitrag der katholischen Presse im Kampf um soziale und wirtschaftliche Befreiung wird vorrangig sein, ebenso die Förderung gerechter menschlicher Beziehungen.

Bei dieser gewaltigen Aufgabe, vor der die katholische Presse steht, muß die Achtung vor der Freiheit von Meinung und Information streng gewahrt bleiben. So wird ein Höchstmaß religiöser Information und Kommentare verbreitet und dem Gesamtkonzept der Entwicklung authentisch christlicher Geist und Wert vermittelt.

Bei der sehr angespannten finanziellen Lage, in der sich die katholische Presse in den meisten Entwicklungsländern befindet, lohnt sich die Mühe, eine gemeinsame ökumenische Presse anzustreben. Multi-Media Zambia ist ein sehr gutes Beispiel eines Versuchs dieser Art, der nicht nur das Image der Christen verbessert, sondern auch dem Fortschritt und der Wirtschaft des Landes geholfen hat. Es ist noch zu früh zu sagen, ob ein solches Wagnis auch in Ceylon möglich sein wird, aber in einer Zeit, in der gemeinsame ökumenische Projekte zunehmen, sollte einem solchen Versuch eine echte Chance gegeben werden. 
Die Presseorgane ihrerseits werden sich so einrichten müssen, daß sie nützlichere Werkzeuge der sozialen Kommunikation in den verschiedenen Ländern sein können. In diesem Organisations- und Orientierungsprozeß müssen die Presseorgane natürlich die berechtigten Abstufungen innerhalb des Rahmens einer christlichen Lebensund Weltsicht respektieren.

Der 4. Kongreß der Katholischen Pressevereinigung Lateinamerikas sagte vor zwei Jahren in Brasilien dazu: „Die Verbreitung des christlichen Denkens wird dort Wirklichkeit, wo Information über das Alltagsleben klug, wohlplaziert und in objektiver Darstellung erfolgt. Diese Information sollte von den Kommentatoren angemessen gewertet werden, die das Vertrauen der Leser zu gewinnen verstehen; sie sollten ihnen auch die Mittel an die Hand geben, die es ihnen gestatten, die Aktualität nach christlichen Kriterien zu beurteilen.

Um dieses Ziel zu erreichen, sind neue, wirkungsvolle Agenturen erforderlich, vor allem für den religiösen und katholischen Bereich; sie sollten so organisiert sein, daß sie den Erwartungen der Entwicklungsländer dienen. ${ }^{22}$

Ein lebenswichtiger Bereich von gegenwärtig höchster Bedeutung, in dem der Presse eine besondere Rolle zufällt, sind die studentischen Unruhen. Die Presse muß alles tun, um die Sympathie der jüngeren Generation zu gewinnen, und ihre Ansichten zu allem, was sie unmittelbar berührt und für sie wichtig ist, reflektieren.

Die katholische Presse von Ceylon insbesondere steht einigen großen Problemen gegenüber, die, wenn sie jetzt nicht zufriedenstellend gelöst werden, mit Sicherheit größere und komplexere Probleme im Verlauf der siebziger Jahren schaffen. Ich will hier nur zwei davon behandeln:

1. Kann die katholische Presse bei den ständig wachsenden Produktionskosten wirtschaftlich überleben?

2. Kann sie sich erfolgreich gegenüber der weltlichen Presse behaupten, die zunehmendes Interesse an der Verbreitung religiöser Nachrichten zeigt?

Die Kostenkrise ist das drängendste und schärfste Problem.

Bei dem steilen Anstieg der Produktionskosten und dem Mangel an Zeitungspapier müssen die katholischen Organe das Problem lösen, indem sie entweder den Verkaufspreis erhöhen oder die Anzeigenkosten heraufsetzen. Aber wie lange kann das so weitergehen?

Das zweite Hindernis - der Wettbewerb mit der weltlichen Presse - ist schwieriger $\mathrm{zu}$ nehmen. Bis vor wenigen Jahren hatte die weltliche Presse der religiösen Nachricht vergleichsweise wenig Raum zur Verfügung gestellt. In den letzten Jahren allerdings hat sich diese Situation völlig umgekehrt. Die weltliche Presse hat heute ein lebhaftes Interesse an Nachrichten aus der katholischen Welt. Die Folge ist, daß Nachrichten von unmittelbar katholischem Interesse schon in der Tagespresse erschienen sind, bevor das katholische Wochenblatt sie an den Mann bringt. Die religiöse Zeitung wird Mitte der Woche gedruckt - in Ceylon normalerweise am Donnerstagabend -, wird aber, wegen der veralteten Vertriebsorganisation, nicht vor dem nächsten Sonntag an die Leute verkauft. Nun ist aber nichts so unerwünscht, als daß Neuigkeiten schal werden. Der lange $Z$ wischenraum von drei Tagen (und manchmal mehr) $z$ wischen Druck und Verkauf der katholischen Zeitung verlangt vom Herausgeber den Verzicht auf echte Aktualität.

Die katholische Tageszeitung (lange in verschiedenen Kreisen diskutiert und vielleicht die einzige radikale Lösung des Problems) wird ein Wunschtraum bleiben müssen. 
Eben wegen dieses gewaltigen Nachteils, daß die katholische Presse in ihrem eigenen Bereich von der weltlichen Presse übertroffen wird, muß die katholische Zeitung in der Zukunft grundsätzlich ihre Einstellung ändern. Sie wird sich stets stärker auf Meinung und Kommentar verlegen müssen als auf die Nachricht selbst. Aber keine Wochenzeitung kann andererseits, wenn sie ankommen will, Nachrichten überhaupt vernachlässigen. Deshalb wird es mehr darauf ankommen, die Nachrichten "auszuschlachten “. Dafür ist die Arbeit von wirklich kompetenten und angesehenen Journalisten wesentlich. Aber diese werden von der katholischen Presse nicht angezogen, weil man ihnen nicht das bezahlen kann, was die weltliche Presse bietet. Die Notwendigkeit, sich mehr und mehr auf „Meinungs-Journalismus" zu konzentrieren, auf mehr analytische und Background-Stories, wird, zusammen mit der Aufgabe, eigene Nachrichtenstories anzuregen, den Herausgebergremien der katholischen Zeitungen neue Lasten aufbürden.

Gleichzeitig hängt die erfolgreiche Durchführung dieser Aufgabe sehr weitgehend von der Handlungsfreiheit ab, die der katholischen Presse in den Entwicklungsländern noch fehlt.

Ein klar umgrenztes Arbeitsgebiet für die Rolle der Kirche in den Entwicklungsländern ist die Presse für Kinder und Schüler. ${ }^{3}$ Oberall in der Welt, vor allem im Osten, nimmt die Zahl der jungen Menschen im Schulalter sprunghaft zu. Automatisch vermehren sich die Unterhaltungsmittel für die Freizeit. Es weist alles darauf hin, daß Kinder und Jugendliche an einer Presse interessiert sind, die älter ist als sie selbst.

Man hat festgestellt, daß drei Phänomene die Entwicklung der Jugendpresse kennzeichnen:

1. die Typen sind zahlreicher (Comics, Magazine, Versuche mit Informationsblättern);

2. eine größere Spezialisierung nach Alter und Publikum;

3. diese Presse gewinnt eine größere kommerzielle Dimension. Junge Leser - das muß nicht betont werden - bilden einen ernstzunehmenden Verbrauchertyp.

Von diesen Grundlagen her hat eine Gruppe von 15 Herausgebern und Erziehern im Westen Schlußfolgerungen erarbeitet, die auch wir nützlicherweise beachten sollten; einige von ihnen glaubten, daß es eine separate und ausschließliche Presse für Jugendliche geben solle, andere meinten, daß spezielle Abteilungen für die Jugend in den Erwachsenenzeitungen geeigneter seien. In einem Punkt aber waren sich alle einig: jungen Leuten sollten nach Technik, Darstellung usw., nur wertvolle Beiträge angeboten werden, und sie sollten von Berufsjournalisten gemacht sein.

Abgesehen von der rein katholischen Presse - muß die Machbarkeit christlicher Zeitungen mehr als bisher erforscht werden. Tatsächlich gibt es gewaltige Möglichkeiten in dieser Richtung. Ein gemeinsames christliches Bemühen, eine Zusammenlegung aller erreichbaren Kräfte und die Schaffung einer christlichen Presse (die das katholische Element miteinschließt) sind wert, näher untersucht zu werden.

Von nicht geringer Bedeutung in der Presse der Entwicklungsländer ist die wirkliche Notwendigkeit, bleibende Institutionen für eine geeignete Journalistenausbildung zu planen, um die sich entwickelnde christliche Presse der Zukunft zu versorgen.

Das Philippine Press Institute zum Beispiel wurde nach dem Vorbild des erfolgreichen American Press Institute an der Columbia University ausgerichtet.

Vor allem südostasiatische Länder brauchen solche Institute, und es müssen Möglichkeiten geschaffen werden, die Journalisten in ihrem Fachbereich auszubilden. Solche 
Institute werden Talente, Modelle und neue Ideen für die rasche Verbesserung des Journalismus in den Entwicklungsländern aufbringen.

Der Bildjournalismus wird seine Auswirkungen auf die Presse der Entwicklungsländer haben.

Die Rolle der Kirche in diesen Dingen größter Dringlichkeit kann nicht überbetont werden. Aber wie steht es dann mit der Zukunft? Ohne Zweifel kann die Zukunft nicht so trostlos sein, wie es scheinen mag, wenn die Kirche sich über die Zeitungen mutig aufs hohe Meer des gedruckten Wortes hinauswagt.

\section{Anmerkungen:}

1. Tarzie Vittachi, Newspaper Revolution, in: "The Asian Magazine ${ }^{\alpha}$, v. 11. 11. 1962, S. 2.

2. "Fourth Congress of Latin American Catholic Union of the Press", in: „Journalistes Catholiques", Paris, Juli-Okt. 1969, S. 27.

3. „Press for Children and Young People ${ }^{\alpha}$, in: „Journalistes Catholiques ${ }^{\alpha}$, Paris, Jan.-April 1969, S. 23.

\section{S U M M A R Y}

The trend toward the public press is becoming noticeable in Ceylon, as in other developing Asian countries. The newly literate must be taken seriously; they are hardly oriented toward the West, but politically alert and alive. The Church's press will have to take this into account. It is a question of imparting the Christian spirit with full respect for freedom of thought. During the current strained financial situation one should consider a common, ecumenical press a genuine opportunity. Over against the secular daily press, which shows increasing interest in the dissemination of current religious news and appears daily, the Catholic weekly - since the Catholic daily remains a wish-dream - will have to be a genuine opinion press, which comments on news in its context. Special attention must also be devoted to the youth sector, which is predominant also in the developing world. These tasks can be mastered only by professional journalists. Therefore centers for the training of journalists will have to be set up. The task of the Catholic press cannot be overestimated.

\section{RESUMEN}

La inclinación hacia los medios de comunicación social es notable tanto en Ceylon como en los restantes países asiáticos en vías de desarrollo. Es necesario tomar en serio a los nuevos lectores, los cuales, aunque poco occidentalizados, están políticamente muy despiertos y conscientes. La prensa de la Iglesia deberá pensar en ellos. Se trata de comunicar el espíritu cristiano con pleno respecto de la libertad de opinión. En la tensa situación financiera debería verse una oportunidad para una ecuménica prensa común. Frente a la prensa mundial secular, que muestra un creciente interés por la difusión de noticias religiosas actuales y que aparece cada día de nuevo, el semanario católico - los diarios católicos son siempre un sueño - debe ser una genuina prensa de opinión que comente las noticias en conjunto. En el mundo del desarrollo hay que atribuir atención especial al sector, cada día más importante, de la juventud. Las tareas pueden ser llevadas a cabo sólo por periodistas profesionales. De aquí la necesidad de crear centros de formación de periodistas. La tarea periodística de la Iglesia no será nunca estimada suficientemente. 